Claremont Colleges

Scholarship@ Claremont

All HMC Faculty Publications and Research

HMC Faculty Scholarship

$1-1-2010$

\title{
Direct Measurements of Island Growth and Step- Edge Barriers in Colloidal Epitaxy
}

Rajesh Ganapathy

Cornell University

Mark R. Buckley

Cornell University

Sharon J. Gerbode

Harvey Mudd College

Itai Cohen

Cornell University

\section{Recommended Citation}

R. Ganapathy, M. Buckley, S. J. Gerbode, and I. Cohen, "Direct measurements of island growth and step-edge barriers in colloidal epitaxy." Science 22, 445-448 (2010). doi: 10.1126/science.1179947

This Article - postprint is brought to you for free and open access by the HMC Faculty Scholarship at Scholarship @ Claremont. It has been accepted for inclusion in All HMC Faculty Publications and Research by an authorized administrator of Scholarship @ Claremont. For more information, please contact scholarship@cuc.claremont.edu. 
Rajesh Ganapathy, et al.

Science 327, 445 (2010);

DOI: $10.1126 /$ science.1179947

This copy is for your personal, non-commercial use only.

If you wish to distribute this article to others, you can order high-quality copies for your colleagues, clients, or customers by clicking here.

Permission to republish or repurpose articles or portions of articles can be obtained by following the guidelines here.

The following resources related to this article are available online at www.sciencemag.org (this information is current as of January 21, 2010):

Updated information and services, including high-resolution figures, can be found in the online version of this article at:

http://www.sciencemag.org/cgi/content/full/327/5964/445

Supporting Online Material can be found at:

http://www.sciencemag.org/cgi/content/full/327/5964/445/DC1

This article cites 25 articles, 6 of which can be accessed for free:

http://www.sciencemag.org/cgi/content/full/327/5964/445\#otherarticles

This article has been cited by 1 articles hosted by HighWire Press; see:

http://www.sciencemag.org/cgi/content/full/327/5964/445\#otherarticles

This article appears in the following subject collections:

Materials Science

http://www.sciencemag.org/cgi/collection/mat_sci 
13. G. B. Partridge, W. Li, R. I. Kamar, Y.-A. Liao, R. G. Hulet, Science 311, 503 (2006); published online 22 December 2005 (10.1126/science. 1122876)

14. L. Luo, B. Clancy, J. Joseph, J. Kinast, J. E. Thomas, Phys. Rev. Lett. 98, 080402 (2007).

15. L. Luo, J. E. Thomas, J. Low Temp. Phys. 154, 1 (2009).

16. S. Riedl et al., Phys. Rev. A 78, 053609 (2008).

17. J. T. Stewart, ]. P. Gaebler, C. A. Regal, D. S. Jin, Phys. Rev. Lett. 97, 220406 (2006).

18. W. Ketterle, M. W. Zwierlein, Riv. Nuovo Cimento $\mathbf{3 1}$, 247 (2008).

19. T.-L. Ho, Phys. Rev. Lett. 92, 090402 (2004).

20. T.-L. Ho, E. J. Mueller, Phys. Rev. Lett. 92, 160404 (2004).
21. S. Inouye et al., Nature 392, 151 (1998).

22. M. Bartenstein et al., Phys. Rev. Lett. 94, 103201 (2005).

23. H. Hu, P. D. Drummond, X.-J. Liu, Nat. Phys. 3, 469 (2007).

24. A. Bulgac, ]. E. Drut, P. Magierski, Phys. Rev. Lett. 99 120401 (2007)

25. Materials and methods are available as supporting material on Science Online.

26. P. Capuzzi, P. Vignolo, F. Federici, M. P. Tosi, Phys. Rev. A 73, 021603 (2006).

27. ]. Joseph et al., Phys. Rev. Lett. 98, 170401 (2007).

28. H. Hu, X.-J. Liu, P. D. Drummond, Phys. Rev. A 73, 023617 (2006).

29. E. Burovski, N. Prokof'ev, B. Svistunov, M. Troyer, Phys. Rev. Lett. 96, 160402 (2006).
30. R. Haussmann, W. Rantner, S. Cerrito, W. Zwerger Phys. Rev. A 75, 023610 (2007).

31. A. Bulgac, ]. E. Drut, P. Magierski, Phys. Rev. A 78, 023625 (2008)

32. We thank Y. Inada and S. Pradhan for experimental assistance. S.N. acknowledges support from the Japan Society for the Promotion of Science.

\section{Supporting Online Material}

www.sciencemag.org/cgi/content/full/327/5964/442/DC1

Materials and Methods

Fig. S1

References

7 October 2009; accepted 24 November 2009

$10.1126 /$ science. 1183012

\section{Direct Measurements of Island Growth and Step-Edge Barriers in Colloidal Epitaxy}

\author{
Rajesh Ganapathy, ${ }^{\star} \dagger$ Mark R. Buckley, Sharon J. Gerbode, Itai Cohen
}

Epitaxial growth, a bottom-up self-assembly process for creating surface nano- and microstructures, has been extensively studied in the context of atoms. This process, however, is also a promising route to self-assembly of nanometer- and micrometer-scale particles into microstructures that have numerous technological applications. To determine whether atomic epitaxial growth laws are applicable to the epitaxy of larger particles with attractive interactions, we investigated the nucleation and growth dynamics of colloidal crystal films with single-particle resolution. We show quantitatively that colloidal epitaxy obeys the same two-dimensional island nucleation and growth laws that govern atomic epitaxy. However, we found that in colloidal epitaxy, step-edge and corner barriers that are responsible for film morphology have a diffusive origin. This diffusive mechanism suggests new routes toward controlling film morphology during epitaxy.

E pitaxy, which is the layer-by-layer growth of a crystalline film on a substrate (1), plays a pivotal role in the fabrication of solid-state and organic semiconductor devices, the creation of strain relief nanostructured arrays, and the design of coatings with novel optical and mechanical properties (2). Understanding the microscopic details of the various growth processes at work continues to be a central focus of surface- and materials-science research (1-6). More recently, this area of research has also branched out to include the self-assembly of nano- and microscale particles into crystalline thin films for the purpose of creating tailor-made metamaterials and photonic band-gap structures (7-11). A variety of experimental techniques have been developed to study atomic homoepitaxy $(1,12)$, but these tools are

Department of Physics, Cornell University, Ithaca, NY 14853, USA.

*To whom correspondence should be addressed. E-mail: rajeshg@jncasr.ac.in

†Present address: International Centre for Materials Science, Jawaharlal Nehru Center for Advanced Scientific Research, Bangalore, Karnataka 560064, India. not appropriate for investigating kinetic pathways in epitaxy of nano- and microscale particles. Without knowledge of these kinetic pathways, kinetic Monte Carlo (KMC) simulations cannot
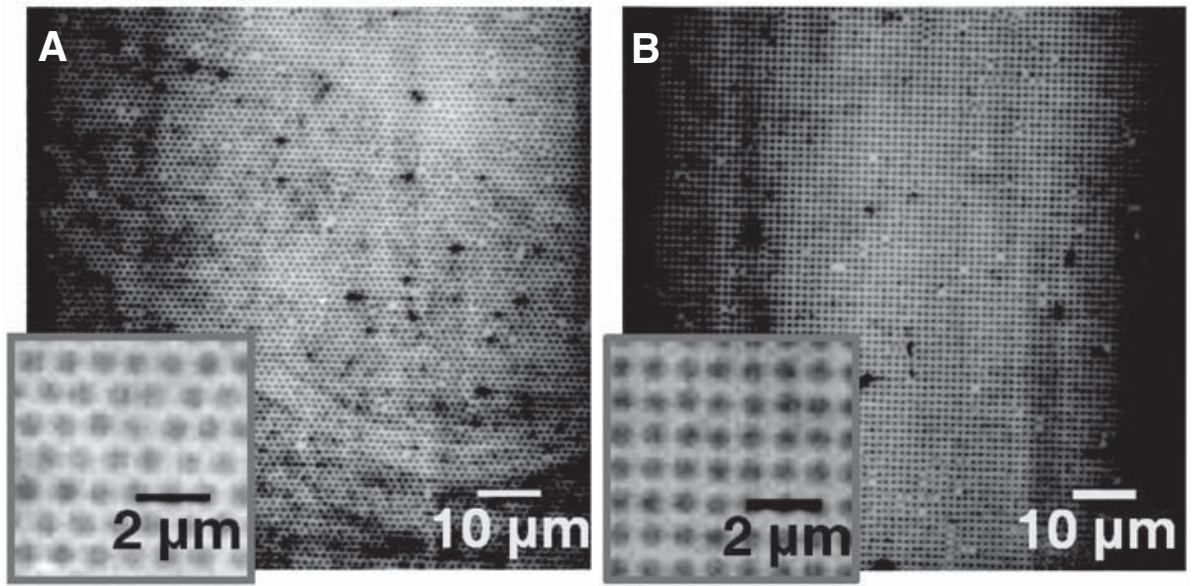

Fig. 1. Confocal micrographs of silica colloid monolayers. The particles are seen as dark circles in a bright fluorescent background. (A) Triangular lattice with lattice spacing of $1.05 \mu \mathrm{m}$. (B) Square lattice with lattice spacing of $1.04 \mu \mathrm{m}$. loids, the barrier originates from the diffusive nature of the particle dynamics. Nevertheless, this effective barrier leads to similar nonuniformities in the 2D and 3D island morphol-

predict accurate growth laws. In fact, at present, it is unclear whether the same kinetic barriers that govern nucleation and growth in atomic systems also govern nano- and microscale particle epitaxy.

We developed and integrated techniques in colloid science to study the epitaxy of microscale particles with an attractive short-range depletion interaction (13). Because colloidal particles can be studied and manipulated at the single-particle level, they are particularly wellsuited for investigating such phenomena. Our experiments show that the two-dimensional (2D) growth laws for atoms and colloids are remarkably similar. In addition, we found that for colloids, there exists an analog of the atomic Ehrlich-Schwoebel barrier (ESB), the energetic cost for moving a particle over a step edge or around an island corner $(14,15)$. In atoms, this barrier is thought to arise from interparticle interactions that are comparable to the atomic spacing. We show that in col- 
ogies. These results demonstrate that key concepts derived from extensive studies on atomic epitaxial growth are directly applicable to film growth of larger-scale particles. In addition, because colloids act as good model systems for studying statistical-mechanics phenomena $(9,10,16-18)$, concepts gleaned from these colloidal deposition experiments should offer insights into atomic and nanoparticle epitaxy.

Our systems consist of charge-stabilized silica or polystyrene colloids with diameters of 1.0 or $1.3 \mu \mathrm{m}$, respectively, and sodium polystyrene sulfonate or sodium carboxyl methyl cellulose polymers with a radius of gyration of about $50 \mathrm{~nm}$. The polymers act as depletants that induce an effective attraction between the particles (13) [supporting online material (SOM) text]. Each colloidal epitaxy experiment consists of sedimenting particles onto a substrate at a fixed flux $F$, which was determined by measuring the area fraction occupied by monomers and islands with time, and with units of monolayers/s (12). To make contact with atomic epitaxy experiments, a single crystalline colloidal monolayer, formed by binding particles to a lithographically patterned template, was used as the substrate (Fig. 1) (19). The sedimented particles perform a $2 \mathrm{D}$ random walk on the substrate by thermally activated hops and coalesce into crystalline islands (movie $\mathrm{S} 1$ ). We found that the monomer surface diffusion constant $D$ is about 0.01 (lattice constants) $)^{2} / \mathrm{s}$, which is 100 times smaller than the diffusion constant for a free particle in liquid (SOM text). This decrease arises from depletioninduced bonds with the underlying substrate, creating an energetic barrier that must be overcome for particles to hop from one interstitial site to another. The ratio of $D / F$ determines the size of the region explored by the particle before it meets another particle, island, or step edge, and this ratio is a key parameter that governs thin-film growth $(1,12)$.

To compare epitaxy of colloids and atoms, a significant overlap in $D / F$ values is essential. The expression for $D$ has an activated form $D=D_{0} \exp \left(-U / k_{\mathrm{B}} T\right)$, where $D_{0}$ is the attempt frequency, $U$ is the activation barrier, $k_{\mathrm{B}}$ is Boltzmann's constant, and $T$ is temperature $(1,12)$. In colloid experiments, $U$ can be made arbitrarily small by tuning the depletant concentration. Thus, even though $D_{0}$ is approximately $10^{-8}$ times as large as in atoms, by careful control over the deposition flux, $D / F$ can be tuned from $10^{-1}$ to $10^{4}$. For atomic deposition, $10^{-1}<D / F<10^{9}$ (12). This significant overlap in $D / F$ values allows for quantitative comparison of the mechanisms governing film growth in these two systems.

Snapshots of nucleation and island growth on a square lattice with $D / F=1300 \pm 100$ are shown in Fig. 2A, 1 to 4 . In our experiments, dimers act as stable nucleation sites. Therefore, we define an island as a cluster equal to or greater than two particles. With time, we observed nucleation of numerous disconnected islands that grew and eventually coalesced to form a single monolayer. The number of disconnected islands per unit area, $n$, versus area fraction of islands, $\Theta$, for this $D / F$ ratio is shown by the solid blue squares in Fig. 2B. Initially, the monomer density on the substrate rises and leads to a linear increase in $n$ (Fig. 2A, 1 and 2, and movie S2). With further increase in the monomer density, $n$ saturates at a critical density $n_{\mathrm{c}}$, because arriving monomers diffuse to nearby islands before encountering other monomers. At later times, existing islands grow in size, leading to coalescence and a decrease in $n$. By varying the substrate symmetry (movie S3), depletant concentration, and bulk colloid volume fraction, we were able to tune $D$ and $F$ independently. This allows for conducting epitaxy experiments at different $D / F$ ratios (Fig. 2B).

We compared the measured $n_{\mathrm{c}}$ versus $D / F$ for our colloid experiments (Fig. 2C, solid symbols) with those from atomic experiments (inverted open triangles) (20), atomic KMC simulations (open squares) (12), rate equation with post deposition mobility (brown line), and the rate equation for stable islands (green line) (20). We found quantitative agreement between the colloid and atom data. At a high $D / F$ ratio, our data approach the $n_{\mathrm{c}} \propto(D / F)^{-1 / 3}$ scaling predicted by classical nucleation theory for systems where dimers form stable islands $(12,20)$. At $D / F \approx 50, n_{\mathrm{c}}$ reaches a maximum and decreases for lower $D / F$ ratios (green line), because diffusion becomes slow and fewer islands nucleate and grow during deposition $(20,21)$. We found that the measured saturation value for $n_{\mathrm{c}}$ agrees with the atomic experiments and the theoretical prediction for systems with stable dimer islands. Finally, we found that the average size of 2D islands increases with increasing $D / F$ (SOM text), and this behavior is consistent with the trend observed in atomic epitaxy (12). These data indicate that scaling laws that are relevant for atoms carry over to 2D film growth of larger-scale particles with attractive interactions.

In addition to controlling island growth, controlling island morphology is of central importance in the growth of high-quality crystalline films $(1,22)$. In atoms, the fundamental parameters that determine island morphology in two

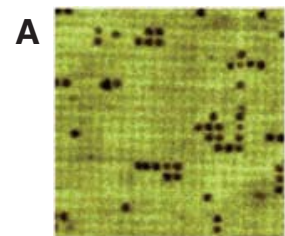

(1) $t=20 \mathrm{hrs}$

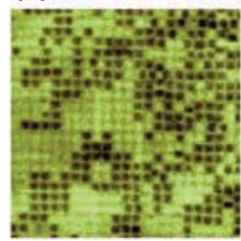

(3) $t=70 \mathrm{hrs}$
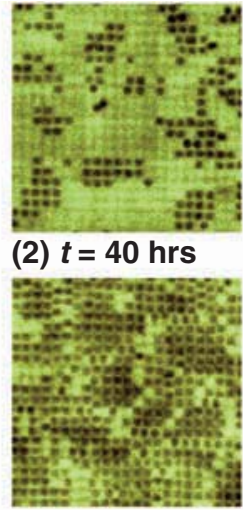

(4) $t=96 \mathrm{hrs}$
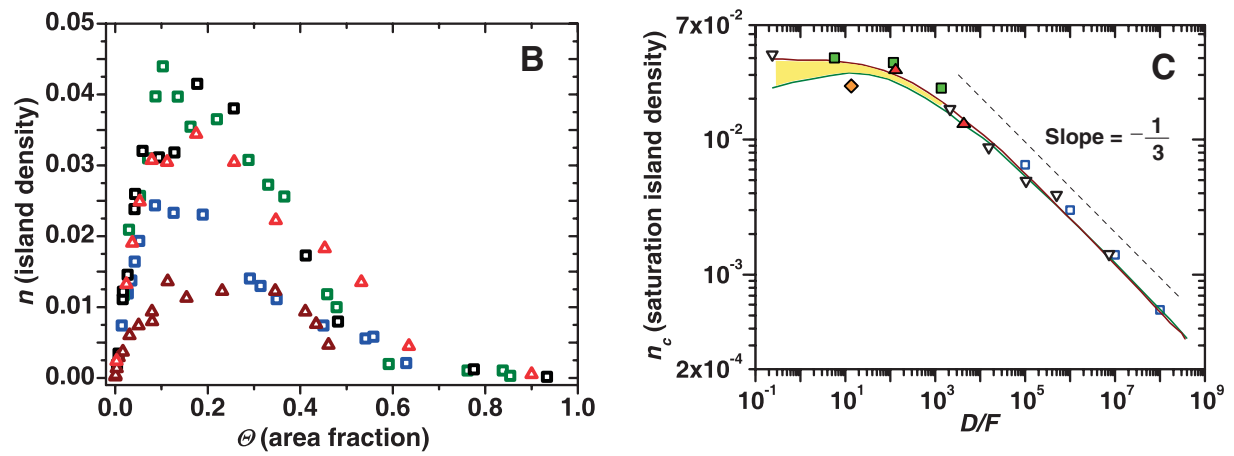

Fig. 2. (A) Four images from an island nucleation and growth experiment on a square template with $D / F=1300 \pm 100$. (B) Island density measurements at various $D / F$ values for square and triangular lattices. Square lattice data for $D / F=1300 \pm 100$ (blue squares), $D / F=116 \pm 8$ (black squares), $D / F=5.7 \pm 0.6$ (green squares). Triangular lattice data for $D / F=$ $4200 \pm 233$ (brown triangles), $D / F=130 \pm 10$ (red triangles). (C) $n_{\mathrm{c}}$ versus $D / F$ for atomic deposition experiments (inverted black triangles); for KMC simulations (blue open squares) [from (12)]; rate equation with post-deposition

mobility is represented by the brown line; rate equation for stable islands is represented by the green line [from $(20,21)]$. The colloid epitaxy experiments with silica particles on the square lattice are shown by green solid squares, whereas those on the triangular lattice are shown by red solid triangles. $n_{\mathrm{c}}$ is obtained from fitting a cubic polynomial to the $n$ versus $\Theta$ data. An experiment with polystyrene particles on a square lattice is shown by the orange solid diamond. The data correspond to systems where dimers form stable island nuclei. 
and three dimensions are the island-corner and the ESB step-edge kinetic barriers $(1,14,15,23)$, respectively. Such barriers are thought to arise from the interactions between the atoms and their neighbors. Specifically, as an atom hops from one interstitial site to another on the same island, it must break bonds with its nearest neighbors and form bonds at the new site.
Because the range of the interaction in atomic systems extends beyond the size of an atom, new bonds are able to form as the old ones are broken. This minimizes the energetic cost for going through the lower coordination number state during the hop. The distances between sites straddling a step edge or corner are longer than those between other adjacent sites on the island; therefore, the energetic barriers for hopping over step edges and corners are substantially larger (Fig. 3, A and B). Because depletion interactions in our colloidal epitaxy experiments are very short-ranged, extending to only $1 / 20$ of the particle diameter, these arguments do not hold for our system. Nevertheless, in our experiments on colloids, we did find evidence of
Fig. 3. (A) Schematic of an adatom diffusing near an island step edge. (B) Energy landscape for atoms near a step edge. (C) Image of islands growing on the monolayer substrate. (D) Image of islands nucleating on top of islands shown in (C) (red outline). The separation between islands in (C) is $\approx 8.0$ lattice constants, whereas the radius of the islands in (D) is $\approx 2.5$ lattice constants. Such features in atomic systems are associated with a step-edge barrier. (E) Polystyrene colloid diffusing on a colloidal monolayer with triangular symmetry. The trajectory of the colloid during a 180-s interval is shown in red with green dots. (F) Trajectory of a polystyrene colloid diffusing on a three-particle island over $180 \mathrm{~s}$. Island rearrangements in our experiments are rare. (G) Interstitial residence time distribution for a colloidal particle diffusing on a monolayer (top) and for a particle diffusing on a threeparticle island (bottom). (H) Energy landscape for colloids near a step edge. (I) Residence time ratios $\frac{\left\langle\tau_{p}=0,1,2, \text { or } 3\right\rangle}{\left\langle\tau_{p}=0\right\rangle}$ versus $p$. Experiments are shown as black squares. Error bars are on the order of the symbol size and show SEM. Simulations in the strong interaction limit (red circles) and weak interaction limit (green circles) are shown. (J) Experimental mean interstitial residence times on a 15-particle island. Color bars indicate residence time.
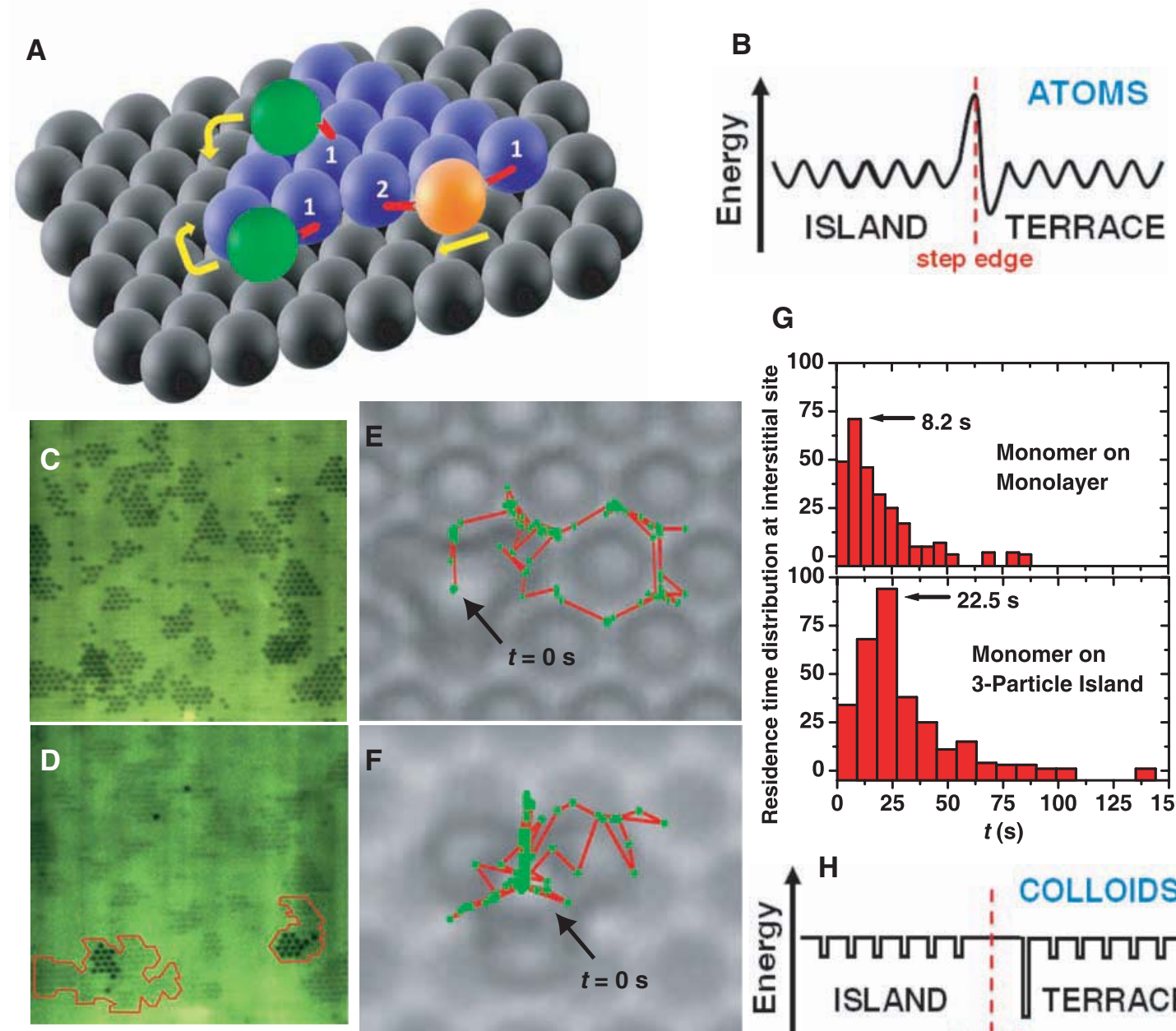

F
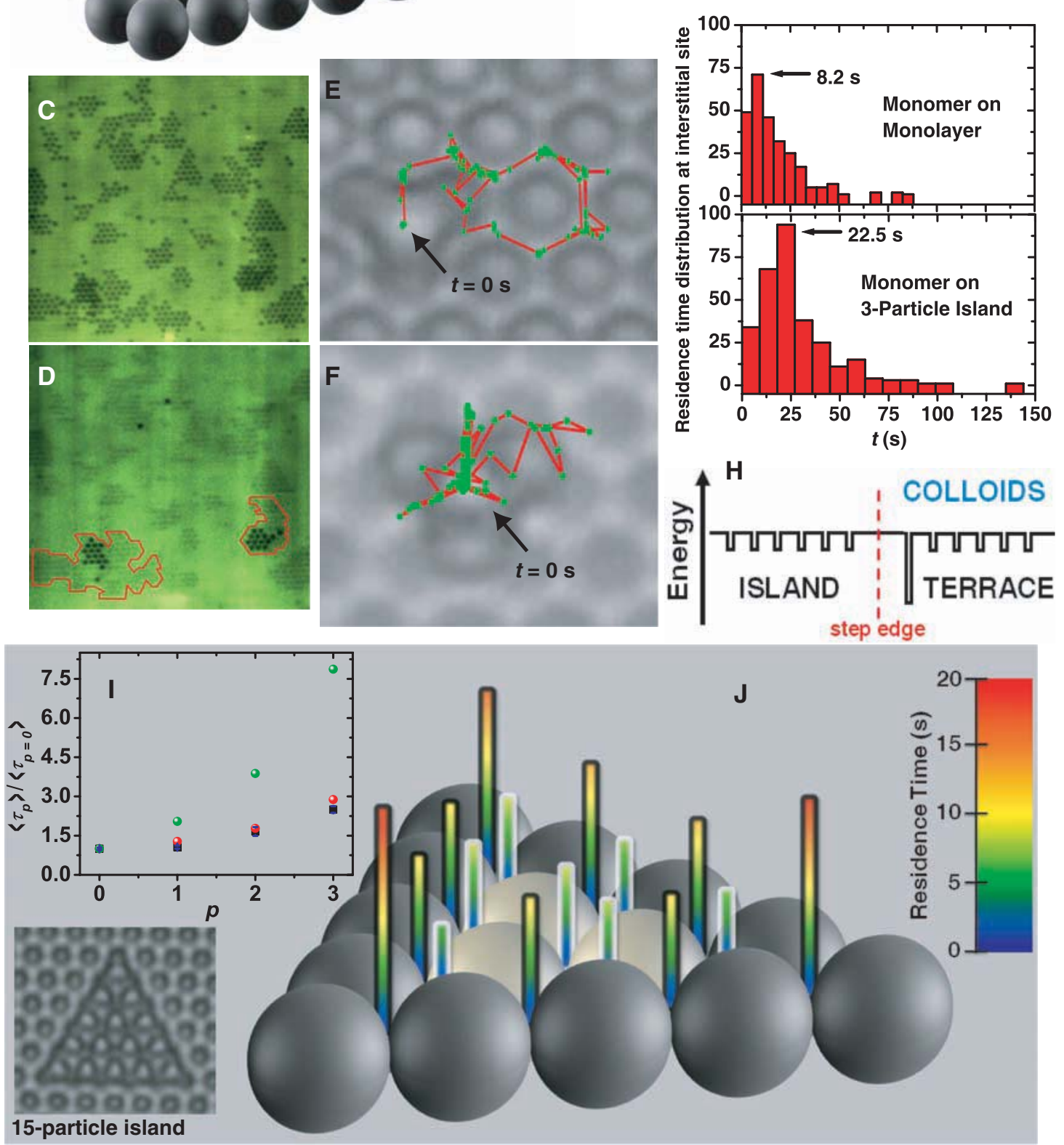
such barriers. For example, we observed nucleation on top of islands even when the separation between them was larger than the average island size. Furthermore, our data are consistent with a decrease in the fractal dimension of 2D islands with $D / F$ (SOM text). These phenomena in atomic epitaxy are associated with ESB step-edge and island-corner barriers (Fig. 3, C and D) (1).

To determine unambiguously whether a stepedge barrier exists for colloidal particles with short-ranged interactions, it is necessary to compare hopping dynamics of particles moving from one island interstitial site to another with those of particles descending island step edges. To quantify these dynamics, we used holographic optical tweezers (Arryx) (24) to place individual colloids on islands consisting of different numbers of particles (SOM text and movie S4). We then turned the tweezers off and tracked the colloids as they moved to different island sites (movies S5 and S6). Particle trajectories of 180-s duration are shown for a large triangular island, where the diffusing colloid explores only interior interstitial sites (Fig. 3E), and a three-particle triangular island, where all excursions are over step edges (Fig. 3F). The trajectories show that island step edges significantly confine the space explored by a diffusing monomer. By repeating these experiments a total of 600 times, we quantified the difference in residence times between interstitial sites away from step edges and those at a step edge. The peak values of the interstitial residence time for a monomer at a step edge is substantially larger than the residence time for a monomer in the island interior (Fig. 3B). This increase indicates that despite the short-range nature of the particle interactions, step-edge barriers also exist in colloidal epitaxy.

The particle trajectories in Fig. 3, E and F, show that once a bond is broken with the underlying lattice, colloid monomers predominantly diffuse along local 1D trajectories corresponding to the valleys formed by the underlying particles (Fig. 3H). Because particles on islands with triangular symmetry must diffuse 2.8 times farther to descend a step edge (SOM text), the diffusion time and the probability of returning to the origin (25) and reforming the original bond increases. Thus, we expect that the mean residence time will increase with the number of longer pathways $p$. To test this prediction, we conducted 100 independent experiments with a total of 1089 monomer moves on a 15-particle triangular island, where different perimeter sites have different $p$ (movie S7). We combined these data with measurements on the large triangular island (Fig. 3E) and three-particle island (Fig. 3F). We plotted the ratios of the mean residence times for sites with $p=0,1$, 2 , or 3 to the mean residence time on an interior site (Fig. 3I) and the mean residence times for all sites on the 15-particle island (Fig. 3J). In accordance with the prediction, we found that the mean residence time increases with $p$.

Depending on the strength of the depletion interaction, there are two limiting regimes. In the weak-interaction limit, the bond breaking time is small and the time taken to diffuse between sites is the dominant contribution to the mean residence time. For diffusion, the mean-squared displacement increases linearly with time. Therefore, the mean residence time scales as the square of the path length. In the strong interaction limit, the bond-breaking time dominates. For a 1D random walker between partially absorbing boundaries, the number of returns to the origin grows linearly with the path length (26). Because the particle re-forms the bonds upon each return, the mean residence time increases linearly with the path length. To determine whether either limit is appropriate for describing our experiment, we modeled the process of moving from one interstitial site to another as a 1D random walk on three line segments that share a common origin and are terminated by absorbing boundaries (27) (SOM text). We have numerically implemented this model and calculated the mean residence time ratios in both the diffusive and strong-interaction limits. The experimentally observed mean residence time ratios are consistent with the strong interaction limit (Fig. 3I)

Collectively, these results indicate that epitaxy in colloidal systems is remarkably similar to epitaxy in atoms. We have shown that, as with atoms, the $D / F$ ratio dictates the $2 \mathrm{D}$ growth laws. In addition, we have uncovered a novel dynamic mechanism that leads to step-edge and corner barriers even in systems with short-range interactions. Implementing techniques that use gravity or electromagnetic fields to bias the diffusion of particles down step edges would lower the step-edge barrier and lead to substantially smoother films. Such techniques might also be applicable for tuning barriers in nanoparticle and molecular systems. The powerful array of tools that we have brought together to investigate colloidal epitaxy may also be helpful in elucidating mechanisms that have proven difficult to study in atoms, such as dynamic stress relaxation mechanisms in strained layer heteroepitaxy (6). Finally, given the rapid advances in synthesizing micro- and nanoscale colloidal particles with directional interactions (28) and anisotropic shapes (29), we expect that future epitaxy experiments with such particles will lead to a valuable exchange of ideas among the fields of microparticle, nanoparticle, and atomic epitaxy.

\section{References and Notes}

1. Z. Zhang, M. G. Lagally, Science 276, 377 (1997).

2. J. V. Barth, G. Costantini, K. Kern, Nature 437, 671 (2005).
3. B. Lewis, D. S. Campbell, J. Vac. Sci. Technol. 4, 209 (1967).

4. T. Michely, ]. Krug, Islands, Mounds, and Atoms: Patterns and Processes in Crystal Growth Far from Equilibrium (Springer-Verlag, Berlin, 2004).

5. J. A. Venables, Philos. Mag. 27, 697 (1973).

6. C. Teichert, Phys. Rep. 365, 335 (2002).

7. A. van Blaaderen, R. Ruel, P. Wiltzius, Nature 385, 321 (1997).

8. Z. Cheng, W. B. Russel, P. M. Chaikin, Nature 401, 893 (1999).

9. P. Schall, I. Cohen, D. A. Weitz, F. Spaepen, Science 305, 1944 (2004).

10. M. E. Leunissen et al., Nature 437, 235 (2005).

11. E. V. Shevchenko, D. V. Talapin, N. A. Kotov, S. O'Brien, C. B. Murray, Nature 439, 55 (2006).

12. H. Brune, Surf. Sci. Rep. 31, 121 (1998)

13. S. Asakura, F. Oosawa, J. Chem. Phys. 22, 1255 (1952).

14. G. Ehrlich, F. G. Hudda, J. Chem. Phys. 44, 1039 (1966).

15. R. L. Schwoebel, E. J. Shipsey, J. Appl. Phys. 37, 3682 (1966).

16. A. M. Alsayed, M. F. Islam, ]. Zhang, P. J. Collings, A. G. Yodh, Science 309, 1207 (2005).

17. J. R. Savage, D. W. Blair, A. J. Levine, R. A. Guyer, A. D. Dinsmore, Science 314, 795 (2006).

18. P. Schall, I. Cohen, D. A. Weitz, F. Spaepen, Nature 440, 319 (2006).

19. Materials and methods are available as supporting material on Science Online.

20. H. Brune, G. S. Bales, J. Jacobsen, C. Borgano, K. Kern, Phys. Rev. B 60, 5991 (1999).

21. In atomic epitaxy, scanning tunneling microscope measurements are conducted after deposition. Thus the remaining monomers continue to diffuse, and this leads to further island growth and/or nucleation. In our colloidal epitaxy measurements, we measured the instantaneous number density of stable islands, and we expect our data to follow the rate equation that ignores post-growth and/or nucleation.

22. J. W. Evans, P. A. Thiel, M. C. Bartelt, Surf. Sci. Rep. 61, 1 (2006).

23. G. Hlawacek et al., Science 321, 108 (2008).

24. E. R. Dufresne, D. G. Grier, Rev. Sci. Instrum. 69, 1974 (1998).

25. G. Polya, Math. Ann. 83, 149 (1921).

26. S. Redner, A Guide to First Passage Processes (Cambridge Univ. Press, Cambridge, 2001).

27. M. A. El-Shehawey, J. Phys. Math. Gen. 33, 9005 (2000).

28. L. Hong, S. Jiang, S. Granick, Langmuir 22, 9495 (2006).

29. V. N. Manoharan, M. T. Elsesser, D. J. Pine, Science 301, 483 (2003).

30. We thank L. Ristroph, J. Savage, T. Arias, ]. Machta, and A. Woll for useful discussions. We would like to especially thank ]. Sethna for helping us set up the numerical calculations for residence times. This research was supported by grants from the NSF Division of Materials Research, the Cornell NanoScale Science and Technology Facility, and in part by award no. KUS-C1-018-02 from King Abdullah University of Science and Technology (KAUST)

\section{Supporting Online Material}

www.sciencemag.org/cgi/content/full/327/5964/445/DC1

Materials and Methods

SOM Text

Figs. $S 1$ to $S 6$

Movies $\mathrm{S} 1$ to $\mathrm{S} 7$

References

30 July 2009; accepted 24 November 2009

10.1126/science. 1179947 